\title{
Comparison of active vs. expectant management of the third stage of labor in women with low risk of postpartum hemorrhage: a randomized controlled trial
}

\author{
Dogukan Yildirim ${ }^{1}$, Sefik E. Ozyurek ${ }^{2}$, Ali Ekiz ${ }^{1}$, Elif C. Eren ${ }^{3}$, Derya Uyan Hendem ${ }^{4}$, \\ Olgu Bafali ${ }^{4}$, Kerem D. Seckin ${ }^{4}$ \\ ${ }^{1}$ Kanuni Sultan Suleyman Education and Research Hospital, Istanbul, Turkey \\ ${ }^{2}$ Bagcilar Education and Research Hospital, Bagcilar, Istanbul, Turkey \\ ${ }^{3}$ Medipol Hospital, Bagcilar, Istanbul, Turkey \\ ${ }^{4}$ Kanuni Sultan Suleyman Education and Research Hospital, Kucukcekmece, Istanbul, Turkey
}

\begin{abstract}
Objectives: To compare the 'strictly' active management protocol in women with low risk of postpartum hemorrhage using the expectant management protocol with respect to changes in hematologic parameters, uterotonics, blood transfusions, or additional interventions.

Material and methods: A randomized controlled prospective trial in which 934 singleton parturients enrolled; 654 were randomly assigned to the active and mixed management groups. The primary outcome parameter was the reduction in hemoglobin concentrations due to delivery, and the secondary outcome parameters were changes in hemoglobin of more than $3 \mathrm{~g} / \mathrm{dL}(\Delta \mathrm{Hb} \geq 3 \mathrm{~g} / \mathrm{dL}$ ), durations of the third stage of labor, need for additional uterotonic agents, blood transfusions, manual removal of the placenta, and surgical evacuation of retained products of conception.

Results: The mean postpartum hemoglobin concentration was significantly higher $(P=0.04)$ in the active management group with a significantly lower reduction ( $P=0.03$ ). Falls of hemoglobin levels of more than $3 \mathrm{~g} / \mathrm{dL}(\Delta \mathrm{Hb} \geq 3 \mathrm{~g} / \mathrm{dL}$ ) were less common in the active management group though not significantly $(P=0.32)$. The mean duration of the third stage of labor was significantly $(P<0.001)$ shorter in the active management group. There was no significant difference between the two groups with regard to the need for additional uterotonic agents, uterine atony, blood transfusion, manual removal of the placenta, surgical evacuation of retained products of conception, and prolonged third stage of labor.

Conclusions: Although active management of the third stage of labor was associated with higher postpartum hemoglobin levels, it did not influence the risk of 'severe postpartum hemorrhage' in women with low risk of postpartum hemorrhage. Key words: postpartum hemorrhage, active management, expectant management, controlled cord traction, third stage of labor
\end{abstract}

\section{INTRODUCTION}

Approximately 127000 maternal deaths caused by postpartum hemorrhage are reported worldwide annually [1]. Postpartum hemorrhage remains the leading global cause of maternal mortality, accounting for at least one-quarter of all maternal deaths [2]. Adequate management of the third stage of labor is critical due to the fact that postpartum hemorrhage often presents following that stage.
Proper management of the third stage of labor makes it possible to avoid or at least alleviate postpartum hemorrhage. Active and physiologic management (also known as expectant approach) have been defined as the two main types of management protocols. Active management consists in early umbilical cord clamping, the use of uterotonic agents after birth, controlled cord traction, and uterine massage. In contrast, in physiologic management clinicians 
await the signs of placental separation and allow for the placenta to fall by maternal effort or spontaneously, with gravity, and do not use uterotonic agents or clamp the cord until placental expulsion [3]. The choice of the optimal method remains controversial. Although international health organizations recommend the active management protocol, especially in hospital settings, trials which support physiologic management have also been reported [4-8].

A trial from 14 European countries demonstrated the third stage of labor management to have significant international as well as national variations [9]. Although active management is precisely described, it is not always strictly applied, and the one which applied is rather nonspecifically referred to as the 'mixed' management, comprising the physiologic protocol diluted with one or two constituents of an active management [10].

\section{OBJECTIVES}

The aim of our study was to compare the expectant management protocol, as it is applied to women with low-risk of postpartum hemorrhage in our clinic, with the'strictly'active management protocol, primarily with respect to changes in the hematologic parameters. The secondary outcome measures were the need for uterotonics, blood transfusions, or additional interventions (manual removal of the placenta, postpartum curettage).

\section{MATERIAL AND METHODS}

This randomized controlled prospective trial was held at the Istanbul Bakirkoy Maternity and Children Hospital in 2010, when approximately 18000 women gave birth. Local Ethics Committee approved of the study (no: 278), and the ethics standards of the 1975 Declaration of Helsinki as revised in 2000 were followed.

Eligibility criteria were: absence of risk factors for postpartum hemorrhage; gestational age of 36-42 weeks; a singleton pregnancy; live fetus; cephalic presentation; expected fetal birth weight of 2500-4500 gm; maternal age of < 40 years; parity (min.-max.) 0-3.

The exclusion criteria were: acute fetal distress, conversion to abdominal delivery during labor, need for labor augmentation, persistent high blood pressure (> 140/90 mm Hg), placenta previa, ablatio placenta or uterine bleeding of any other cause encountered during pregnancy or labor; previous C-section; uterine scar; postpartum hemorrhage in previous pregnancies; hydramnios; symptoms of maternal infection; drug use in labor; abnormal placentation (accreta, increta or percreta), coagulation defects, forceps or vacuum extraction, hemoglobin concentration of $<8 \mathrm{~g} / \mathrm{dL}$; use of anticoagulants and tocolytics during pregnancy, multiple gestations, any known uterine malformations, and deep vaginal lacerations.
The effect of the tested protocol on the reduction in hemoglobin concentrations due to delivery was the primary outcome measure of this study. In our hospital, mean change in hemoglobin concentrations due to delivery is $-1.3 \mathrm{~g} / \mathrm{dL}$ using the expectant management protocol in the third stage of labor. We tested the hypothesis that 'active management should reduce the mean reduction in hemoglobin levels due to delivery by $50 \%$. At a power level of $80 \%$, the minimum number of patients required was 310 patients in the 2 groups. Nine hundred thirty-four parturients were initially eligible in the stated time period. The women consented and were enrolled for the study at the time of admission for labor and delivery. We prepared standard forms assigned for each group; half were tagged with 'active management', and the other half with 'expectant management'. Allocation concealment was made using sealed opaque envelopes and took place when vaginal delivery was imminent, at the end of the second stage of labor.

The 'active management of the third stage' group was managed in accordance with the recommendations of the World Health Organization (WHO, 2007): 10 IU oxytocin intramuscular injection within the first minute after delivery, early umbilical cord clamping, and application of the controlled cord traction with uterine massage. The 'expectant management of the third stage' group was managed as: umbilical cord clamping after cord pulsation had slowed down, placental separation signs were expected (a sudden gush of blood from the vagina, lengthening of the umbilical cord into a lower segment, rise of the uterus in the abdomen); the placenta was allowed to fall by maternal effort and gravity; and a 10 IU oxytocin IM injection was administered after placental expulsion.

We ordered a complete blood count from all patients at the onset of labor to report the prepartum hemoglobin and hematocrit levels, and measured the duration of the third stage of labor. The placenta was removed manually if it did not fall after 30 minutes. In both groups, uterine massage was performed every 15 minutes until leaving the delivery room. If vaginal bleeding was persistent, the patient was examined vaginally to check for overlooked lacerations of the birth tract, uterine massage was conducted, and extra uterotonics were administered in the event that these measures were not sufficient. The proportion of patients who required extra uterotonics or additional interventions was recorded in the two groups for comparison. A complete blood count was ordered for all patients 24 hours postpartum and hemoglobin-hematocrit levels were recorded. Our policy was to refrain from postpartum blood transfusions unless the hemoglobin concentrations were lower than $6 \mathrm{~g} / \mathrm{dL}$, or the patient displayed symptoms and signs of hypoxemia while the hemoglobin concentration was lower than $8 \mathrm{~g} / \mathrm{dL}$ at the end of the $24^{\text {th }}$ postpartum hour. 
In order to compare the two groups in terms of severe postpartum hemorrhage, the results were stratified as reductions in hemoglobin levels of more than $3 \mathrm{~g} / \mathrm{dL}$ $(\Delta \mathrm{Hb} \geq 3 \mathrm{~g} / \mathrm{dL})$, and $\Delta \mathrm{Hb}<3 \mathrm{~g} / \mathrm{dL}$ caused by blood loss in the third stage of labor. Other secondary parameters of comparison included prolonged third stage of labor, transfusion requirement, requirement of additional uterotonics, additional interventions including manual removal of the placenta, and the requirement of additional surgery or curettage. SPSS 15.0 for Windows (SPSS Inc, Chicago, IL, USA) was used for statistical analysis. The Kolmogorov-Smirnov test was used to ensure normal distribution and equality variance of continuous variables between the two groups. Student's t-test and non-parametric Mann-Whitney U test were used to analyze normal and non-normal distribution data, respectively. The Chi-square test was used for categorical variables and Fisher's exact test was used if required. A linear regression model was defined to identify independent variables which affected the main output variable, i.e. hemoglobin reduction following delivery in our population. Factor analysis was then used to ensure that the effects of these independent variables were similar in both, the study and control groups. These independent predictors were then used as covariate factors to compare the study and control groups in terms of hemoglobin reduction due to delivery, while minimizing the effects of these covariates using ANCOVA. The $p$-value of $<0.05$ was considered as statistically significant.

\section{RESULTS}

A total of 934 parturients in active labor were enrolled in the trial (Figure 1). Seventy-eight patients were converted to abdominal delivery because of fetal distress, cord prolapse, placental abruption, and persistent bradycardia during the second stage of labor. Labor augmentation was used in 138 patients due to delay in the first stage of labor. Thirty-seven patients later refused to participate in the trial. Five patients experienced bleeding during the first stage of labor, and 7 parturients required instrumental delivery. Some 669 parturients were randomized into the 2 treatment arms: 333 patients in the first group received active management and 336 in the second group were treated with the expectant management protocol. Six patients in the active management group and 9 in the expectant manage-

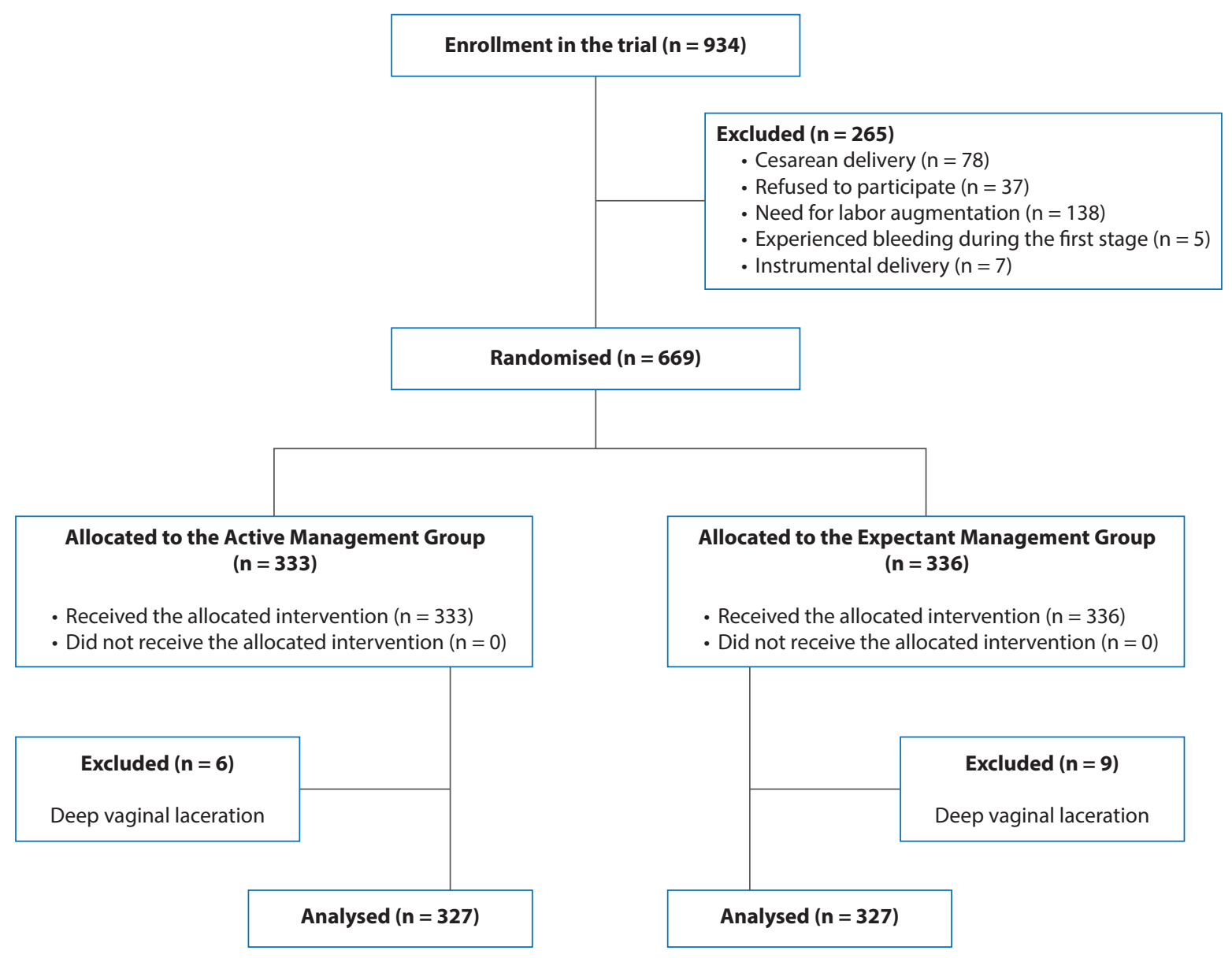

Figure 1. Study flowchart 
Table 1. Clinical features of the groups

\begin{tabular}{|c|c|c|c|}
\hline & $\begin{array}{c}\text { Expectant } \\
\text { Management Protocol } \\
\mathrm{n}=327\end{array}$ & $\begin{array}{c}\text { Active } \\
\text { Management Protocol } \\
n=327\end{array}$ & $\mathbf{P}$ \\
\hline $\begin{array}{l}\text { Age } \\
\text { Mean }(S D)^{\beta}\end{array}$ & $25.92(5.13)$ & $25.98(5.25)$ & 0.88 \\
\hline $\begin{array}{l}\text { Body mass index } \\
\text { Mean }(\mathrm{SD})^{\beta}\end{array}$ & $27.94(3.8)$ & 27.55 (3.39) & 0.17 \\
\hline $\begin{array}{l}\text { Gestational week } \\
\text { Mean }(S D)^{\beta}\end{array}$ & $39.84(1.81)$ & $38.74(1.66)$ & 0.27 \\
\hline $\begin{array}{l}\text { Birth weight }[g] \\
\text { Mean }(S D)^{\beta}\end{array}$ & 3248.9 (315.19) & 3316.39 (258.45) & 0.34 \\
\hline $\begin{array}{l}\text { Length of labor (min.) } \\
\text { Mean (SD) }{ }^{\beta}\end{array}$ & $328.6(262.76)$ & $353.43(282.88)$ & 0.25 \\
\hline $\begin{array}{l}\text { Prepartum hemoglobin }[\mathrm{g} / \mathrm{dL}] \\
\text { Mean }(\mathrm{SD})^{\beta}\end{array}$ & $11.5(1.23)$ & 11.59 (1.32) & 0.38 \\
\hline $\begin{array}{l}\text { Prepartum hematocrit (\%) } \\
\text { Mean (SD) }\end{array}$ & 34.91 (3.48) & 35.04 (3.73) & 0.62 \\
\hline Episiotomy, $\mathrm{n}(\%)^{\gamma}$ & $229(70.03)$ & $226(69.11)$ & 0.8 \\
\hline $\begin{array}{l}\text { Parity, } n(\%)^{\gamma} \\
\text { Nulliparity } \\
\text { Multiparity }\end{array}$ & $\begin{array}{l}131(40.06) \\
196(59.94)\end{array}$ & $\begin{array}{l}129(39.45) \\
198(60.55)\end{array}$ & 0.87 \\
\hline
\end{tabular}

${ }^{\beta}$ Student's T test or Non-parametric tests; ${ }^{\vee C h i-s q u a r e ~ t e s t . ~ S D ~ — ~ s t a n d a r d ~ d e v i a t i o n ~}$

Table 2. Outcome measures of the study groups

\begin{tabular}{|c|c|c|c|c|}
\hline & $\begin{array}{c}\text { Expectant } \\
\text { Management } \\
n=327\end{array}$ & $\begin{array}{c}\text { Active } \\
\text { Management } \\
n=327\end{array}$ & $\begin{array}{l}\text { Odds ratio } \\
\text { Cl: [5-95\%] }\end{array}$ & $\mathbf{P}$ \\
\hline $\begin{array}{l}-\Delta \mathrm{Hb} \text { loss } \geq 3 \mathrm{~g} / \mathrm{dL} \\
\text { Severe postpartum hemorrhage, } \mathrm{n}(\%)^{\gamma}\end{array}$ & $16(4.9)$ & $10(3.1)$ & $0.73-3.64$ & 0.23 \\
\hline $\begin{array}{l}\text { Postpartum } 24^{\text {th }} \text { hour } \mathrm{Hb}[\mathrm{g} / \mathrm{dL}] \\
\text { Mean }( \pm \mathrm{SD})^{\beta}\end{array}$ & $10.09(0.72)$ & $10.78(0.68)$ & - & $0.04^{*}$ \\
\hline $\begin{array}{l}\Delta \mathrm{Hb}[\mathrm{g} / \mathrm{dL}] \\
\text { Mean }( \pm \mathrm{SD})^{a}\end{array}$ & $1.62(0.12)$ & $1.02(0.23)$ & - & $0.03^{*}$ \\
\hline $\begin{array}{l}\text { Postpartum } 24^{\text {th }} \text { hour Hct (\%) } \\
\text { Mean }( \pm S D)^{\beta}\end{array}$ & $30.46(3.98)$ & $30.94(4.08)$ & - & 0.13 \\
\hline $\begin{array}{l}\Delta \operatorname{Hct}(\%) \\
\text { Mean }( \pm S D)^{\beta}\end{array}$ & $4.45(2.89)$ & $4.1(3.05)$ & - & 0.14 \\
\hline $\begin{array}{l}\text { Duration of 3. stage (minutes) } \\
\text { Mean }( \pm S D)^{\beta}\end{array}$ & $9.26(4.59)$ & $4.11(2.32)$ & - & $<0.001 *$ \\
\hline Lengthening of 3 . stage ( $>30$ minutes), $n(\%)^{Y}$ & $3(0.9)$ & $2(0.6)$ & $0.17-3.4$ & 0.65 \\
\hline Additional uterotonic use, $\mathrm{n}(\%)^{\gamma}$ & $30(9.17)$ & $27(8.26)$ & $0.27-1.82$ & 0.68 \\
\hline Manual removal of placenta, $n(\%)^{\gamma}$ & $3(0.92)$ & $2(0.61)$ & $0.16-3.32$ & 0.65 \\
\hline Curettage, $n(\%)^{\gamma}$ & $0(0)$ & $3(0.92)$ & NA & 0.08 \\
\hline Transfusion, $n(\%)^{\gamma}$ & $4(1.2)$ & $3(0.91)$ & $0.3-5.9$ & 0.4 \\
\hline Atony, $\mathrm{n}(\%)^{\mathrm{Y}}$ & $3(0.92)$ & $4(1.22)$ & $0.17-3.34$ & 0.7 \\
\hline
\end{tabular}

${ }^{\beta}$ Student's T test or Non-parametric test; ; ${ }^{\top}$ Chi-square test; ${ }^{~}$ Multivariable linear regression, factor analysis, ANCOVA; ${ }^{*}$ Statistically significant. $\triangle \mathrm{Hb}$ - change in the hemoglobin concentration; $\Delta \mathrm{Hct}$ - change in the hematocrit; $\mathrm{SD}$ - standard deviation; $\mathrm{Cl}$ - confidence interval

ment group were excluded because of deep vaginal lacerations. Thus, 327 patients in the active management group and 327 patients in the expectant management group with low risk for postpartum hemorrhage were analyzed. There were no statistically significant differences between the two groups with regard to basic characteristics (age, gestational week, body mass index, parity, episiotomy, and mean length of labor) (Table 1). 
Table 3. Change in hemoglobin concentrations $\geq 3 \mathrm{~g} / \mathrm{dL}^{(a)}$

\begin{tabular}{|c|c|c|c|}
\hline & $(-)$ & $(+)$ & Total \\
\hline Active Management ${ }^{(\beta)}$ & $\begin{array}{c}317 \\
96.9 \%\end{array}$ & $\begin{array}{c}10 \\
3.1 \%\end{array}$ & $\begin{array}{c}327 \\
100 \%\end{array}$ \\
\hline Expectant Management ${ }^{(\varepsilon)}$ & $\begin{array}{c}311 \\
95.1 \%\end{array}$ & $\begin{array}{c}16 \\
4.9 \%\end{array}$ & $\begin{array}{c}327 \\
100 \%\end{array}$ \\
\hline Total & $\begin{array}{l}628 \\
96 \%\end{array}$ & $\begin{array}{l}26 \\
4 \%\end{array}$ & $\begin{array}{c}654 \\
100 \%\end{array}$ \\
\hline
\end{tabular}

(a) Defining severe hemorrhage; Chi-square: $P=0.32$; no significant difference between the two protocols; ${ }^{(\beta)}$ The active management of the third stage of labor group; ${ }^{(\varepsilon)}$ The mixed management protocol for the third stage of labor group

There were no statistically significant differences in prepartum hemoglobin and hematocrit levels (Table 2). Hemoglobin levels following delivery were higher $(P=0.04)$ in the active management group.

A linear regression model was used to define the main output variable, the hemoglobin reduction following delivery in our population $(P<0.001)$; the independent variables were: gravida ( $B=-0.06 ; P<0.01)$; gestational age, a very slight effect $(B=0.04 ; P=0.02)$; and prepartum hemoglobin levels $(B=0.09 ; P=0.008)$. Factor analysis revealed that the effects of these independent variables were similar in both, the study and control groups. These independent predictors were then used as covariate factors to compare the study and control groups in terms of hemoglobin reduction due to delivery, while disregarding these covariates using ANCOVA. Extracting the effects of these covariates, the choice of management protocol was found to be a significant determining factor in predicting hemoglobin reduction, which was significantly lower in the active management group: (1.02 g/dL \pm 0.23 vs. $-1.62 \mathrm{~g} / \mathrm{dL} \pm 0.12 ; \mathrm{P}=0.03)$.

Whether'severe hemorrhage' could be prevented by the management protocol choice was tested using $\Delta \mathrm{Hb} \geq 3 \mathrm{~g} / \mathrm{dL}$ as the threshold. There was no difference between the two groups (3.1\% vs. 4.9\%; Odds Ratio: 0.61; [0.27-1.37], $\mathrm{P}=0.32$ ) (Table 3). The third stage was shorter, $(9.26( \pm 4.59)$ minutes vs. 4.11 ( \pm 2.32 ) minutes) in the active management group, which was statistically significant $(P=0.001)$ (Table 2$)$. No difference was obtained between the two groups with the additional use of oxytocin, manual expulsion of placenta, curettage, blood transfusion demand, uterine atony, and lengthening of the third stage (> 30 minutes) (Table 2 ). No maternal death or requirement for additional surgery was observed in either of the two groups.

\section{DISCUSSION}

In our study, we found significantly higher mean postpartum hemoglobin levels and lower mean decrements in hemoglobin levels following delivery in the active management group as compared to the expectant management group.
No positive effect in the prevention of severe postpartum hemorrhage was observed. However, it is challenging to define a decrease in an already low baseline incidence for severe postpartum hemorrhage with low-risk patients and limited sample sizes. Our findings did not confer'any preventive effect on the secondary outcome measures of the need for extra uterotonics, blood transfusions, or additional interventions.' To the best of our knowledge, our study has been the first to compare these protocols in a low-risk population.

The active management protocol in our study group differed from the expectant protocol in the control group in 3 aspects: the timing of the uterotonic administration, umbilical cord clamping, and umbilical cord traction. The timing of uterotonic administration was studied by Soltani et al., in a meta-analysis and was not found to be significant with respect to severe postpartum hemorrhage, amount of blood loss, retained placenta, requirement for uterotonics, or transfusions [11]. McDonald et al., analyzed the timing of umbilical cord clamping in a Cochrane review of 11 trials and 2898 patients [12]. Their conclusion was that delaying umbilical cord clamping had no effect on the amount of blood loss or severe postpartum hemorrhage rates, but resulted in higher neonatal hemoglobin levels and increased rates of neonatal jaundice.

With regard to the effect of umbilical cord traction, Gulmezoglu et al., compared active management performed with and without controlled cord traction in a large WHO multi-center trial, which is the largest postpartum bleeding surveillance study conducted to date, comprising 23681 parturients inclusive of all risk subgroups [13]. In the Gulmezoglu study, patients were stratified into two groups according to the treatment protocols; the full package and the simplified packages. In both groups, oxytocin IM was administered just after the delivery of the fetus. Controlled cord traction was applied in the full package group, whereas placental separation was managed expectantly in the simplified package group. The timing of the postpartum oxytocin injection in the expectant management protocol in our study was the only prominent variation from its 'simplified package' counterpart in the Gulmezoglu study, which we made after placental separation. The Gulmezoglu trial only showed a slight increased risk of severe postpartum hemorrhage in cases without controlled cord traction. The study group concluded that the main component of active management was the use of uterotonics and that controlled cord traction could be omitted when skilled birth attendants were not available.

Du et al. [14], in a recent meta-analysis of 5 randomized controlled trials, similarly reported that umbilical cord traction decreased postpartum blood loss. However, it fell short of preventing severe postpartum hemorrhage (> $1000 \mathrm{cc}$ ), a need for additional uterotonics, or transfusions. 
Deneux-Tharaux et al. [15], published a multi-center randomized controlled trial of 4355 patients who were randomly allocated to 2 groups of placental separation with cord traction and standard placental expulsion. The primary outcome measure was the incidence of severe postpartum hemorrhage (>500 mL). The authors concluded that cord traction had no effect. Some secondary outcome measures were improved, however, including a shortened third stage of labor, and a reduced need for manual removal of the placenta.

Begley et al. [3], compared the active and expectant management of the third stage of labor in a Cochrane database meta-analysis. This meta-analysis showed that although active management reduced mean blood loss and postpartum hemorrhage $(>500 \mathrm{cc})$, there was no statistically significant reduction in severe postpartum hemorrhage (> $1000 \mathrm{cc}$ ) for women at low risk for bleeding. Also, hypertension, pain and discomfort, bleeding after discharging the patient, and low birth weight still remained as possible disadvantages of active management.

Our study is not without limitations. Having not directly measured the amount of blood loss, a reduction of more than $3 \mathrm{~g} / \mathrm{dL}(\Delta \mathrm{Hb} \geq 3 \mathrm{~g} / \mathrm{dL}$ ) in hemoglobin levels at 24 hours postpartum was considered to indirectly denote severe postpartum hemorrhage (>1000 ml). Only the analyzer of the outcome data was blinded to the study groups because the performer could not be blinded to the allocated management protocol. We did not have data about neonatal conditions or rates of hospital readmissions.

The need for additional interventions including curettage, manual removal of the placenta, extra uterotonic administration, transfusions or uterine atony rates was similar between the two groups. This may be due to the low rates of these variables in both groups, and the low-risk population being studied. Our data show that besides providing a smaller decrement in hemoglobin concentrations due to delivery, the active management protocol also shortens the duration of the third stage of labor.

Our findings suggest that if active management of the third stage of labor is preferred for low-risk parturients within a level-three care hospital in a developing country, it is not likely to provide any benefit beyond reducing the decrement of hemoglobin concentrations caused by delivery.

\section{Conflict of interest}

The authors declare that they have no conflicts of interest with any third party and that they have no financial disclosure to be made.

\section{REFERENCES}

1. WHO: Reducing the global burden: postpartum hemorrhage. Making Pregnancy Safer. 2007, 1 (4), 8.

2. Khan KS, Wojdyla D, Say L, Gülmezoglu AM, Van Look PF. WHO analysis of causes of maternal death: A systematic review. Lancet. 2006, 367, 1066-1074.

3. Begley CM, Gyte GML, Devane D, McGuire W, Weeks A. Active versus expectant management for women in the third stage of labor. Cochrane Database Syst Rev. 2015, (3), CD007412.

4. WHO: WHO recommendations for the prevention and treatment of postpartum hemorrhage. http://apps.who.int/iris/bitstream/10665/75411/1/9789241548502 eng.pdf,2012.

5. International Confederation of Midwives, International Federation of Gynecology and Obstetrics. Joint statement. Prevention and treatment of postpartum hemorrhage. New advances for low resource settings. Joint statement. http://www.who.int/pmnch/2006/figo2006statementeng. pdf,2006.

6. National Institute for Health and Clinical Excellence: Normal labor: third stage. Intrapartum care: care of healthy women and their babies during childbirth, Guideline 55. London: National Institute for Health and Clinical Excellence, 2007.

7. New Zealand College of Midwives:Third stage management practices of midwife lead maternity carers: an analysis of the New Zealand College of Midwives Midwifery Database Information (2004-2008). Christchurch: New Zealand College of Midwives, 2009.

8. Davis D, Baddock S, Pairman S, [et al.].Risk of Severe Postpartum Hemorrhage in Low-Risk Childbearing Women in New Zealand: Exploring the Effect of Place of Birth and Comparing Third Stage Management of Labor. Birth. 2012, 39 (2), 98-105.

9. Winter C, Macfarlane A, Deneux-Tharaux C, [et al.]. Variations in policies for management of the third stage of labor and the immediate management of postpartum hemorrhage in Europe. BJOG. 2007, 114, 845-854.

10. Harris T. An explanation for third stage practice variation: The theory of contingent decision making; Normal labor and birth: 3rd Research Conference; Grange-over-Sands, England, UK 2006.

11. Soltani $H$, Poulose TA, Hutchon DR. Placental cord drainage after vaginal delivery as part of the management of the third stage of labor. Cochrane Database Syst Rev. 2011, 9.

12. McDonald SJ, Middleton P. Effect of timing of umbilical cord clamping of term infants on maternal and neonatal outcomes. Cochrane Database Syst Rev. 2008, 16 (2).

13. Gülmezoglu AM, Lumbiganon $P$, Landoulsi $S$, [et al.]. Active management of the third stage of labor with and without controlled cord traction: a randomized, controlled, non-inferiority trial. Lancet. 2012, 379 (9827), 1721-1727.

14. DuY, Ye M, Zheng F. Active management of the third stage of labor with and without controlled cord traction: a systematic review and meta-analysis of randomized controlled trials. Acta Obstet Gynecol Scand. 2014, 93 (7), 626-633.

15. Deneux-Tharaux $C$, Sentilhes L, Maillard F, [et al.]. Effect of routine controlled cord traction as part of the active management of the third stage of labor on postpartum hemorrhage: multicenter randomized controlled trial (TRACOR). BMJ. 2013, 346, 1541. 